\title{
Mobile 3-dimensional (3D) C-arm system-assisted transbronchial biopsy and ablation for ground-glass opacity pulmonary nodules: a case report
}

\author{
Junxiang Chen ${ }^{1,2,3}$, Fangfang Xie ${ }^{1,2,3}$, Xiaoxuan Zheng ${ }^{1,2,3}$, Ying $\mathrm{Li}^{1,2,3}$, Shuaiyang Liu $^{1,2,3}$, Kevin C. Ma \\ Taichiro Goto $^{5}$, Tobias Müller ${ }^{6}$, Edward D. Chan ${ }^{7}$, Jiayuan Sun ${ }^{1,2,3}$ \\ ${ }^{1}$ Department of Respiratory Endoscopy, Shanghai Chest Hospital, Shanghai Jiao Tong University, Shanghai, China; ${ }^{2}$ Department of Respiratory \\ and Critical Care Medicine, Shanghai Chest Hospital, Shanghai Jiao Tong University, Shanghai, China; ${ }^{3}$ Shanghai Engineering Research Center of \\ Respiratory Endoscopy, Shanghai, China; ${ }^{4}$ Section of Interventional Pulmonology, Division of Pulmonary, Allergy, \& Critical Care, University of \\ Pennsylvania, Philadelphia, PA, USA; ${ }^{5}$ Lung Cancer and Respiratory Disease Center, Yamanashi Central Hospital, Yamanashi, Japan; ${ }^{6}$ Department of \\ Pneumology and Intensive Care Medicine, University Hospital RWTH Aachen, Aachen, Germany; ${ }^{7}$ Division of Pulmonary Sciences \& Critical Care \\ Medicine, University of Colorado Anschutz Medical Campus, Aurora, Colorado, USA \\ Correspondence to: Jiayuan Sun, MD, PhD. Department of Respiratory Endoscopy, Department of Respiratory and Critical Care Medicine, Shanghai \\ Chest Hospital, Shanghai Jiao Tong University, No. 241 West Huaihai Road, Shanghai 200030, China. Email: xkyyjysun@163.com.
}

\begin{abstract}
Identification of pulmonary ground-glass opacity (GGO) lesions during bronchoscopic procedures remains challenging, as GGOs cannot be directly visualized under 2-dimensional (2D) fluoroscopy and are often difficult to detect by radial endobronchial ultrasound. Recently, a mobile 2D/3D C-arm fluoroscopy system was developed that provides both 2D fluoroscopy and mobile $3 \mathrm{D}$ imaging to assess and confirm the location of the lesions and ancillary bronchoscopic tools. However, previous studies focused mainly on experience of utilizing mobile 3D C-arm system for transbronchial biopsy of solid pulmonary nodules. Here, we evaluated the feasibility of mobile 3D imaging assisted transbronchial biopsy with and without ablation of two patients with GGO nodules. The first patient underwent biopsy only, and the second patient underwent biopsy in the right upper lobe lung nodule and ablation of a left upper lobe lung nodule in one session. Procedures in both patients were successfully performed, and no significant complications have been observed intra- or post-procedurally. Our case study highlights the potential value of the mobile $3 \mathrm{D}$ imaging system in accurate identification of the target lung lesion, confirmation of bronchoscopic tools within the lesion, and assessment of the target lesion and surrounding tissue following bronchoscopic ablation procedure. Furthermore, a "one-stop shop" bronchoscopy workflow combining both biopsy and ablation for one or more lung lesions in one session could be made possible by utilizing a hybrid mobile 2D/3D C-arm system in the bronchoscopy suite.
\end{abstract}

Keywords: Ground-glass opacity (GGO); bronchoscopy; fluoroscopy; mobile 3D C-arm; case report

Submitted May 18, 2021. Accepted for publication Jul 22, 2021.

doi: $10.21037 /$ tlcr-21-561

View this article at: https://dx.doi.org/10.21037/tlcr-21-561

\section{Introduction}

Ground-glass opacity (GGO) is defined as a hazy area of opacification without obscuration of the underlying pulmonary airway and vasculature. GGO may be classified as pure GGO (pGGO) or mixed GGO (mGGO), the latter referring to the presence of a solid component (1). A major concern of GGOs is that they have the significant potential to be a sign of a primary lung cancer, with a reported malignancy rate of $63 \%$ for $\mathrm{mGGO}$ and $18 \%$ for pGGO (2). It is essential to differentiate between benign and malignant GGOs through transbronchial biopsy prior to treatment.

However, identification of GGO remains challenging 
during a bronchoscopic procedure, as visualization of GGOs under 2-dimensional (2D) fluoroscopy is difficult and may lead to suboptimal outcomes (3). In order to improve the localization and access of GGO nodules, multiple navigation technologies, including radial probe endobronchial ultrasound (EBUS), virtual bronchoscopic navigation (VBN), electromagnetic navigation bronchoscopy (ENB) and cone-beam computed tomography (CBCT), as well as other modalities, can be used during bronchoscopy (4). In the EBUS imaging, pGGO is visualized as a blizzard sign while mGGO is usually shown as a mixed blizzard sign; in practice, both of these two patterns are often difficult to be detected by EBUS.

Meanwhile, unlike other navigation techniques that either provide 2D images or use preprocedural CT for pathway planning, CBCT can produce real-time $3 \mathrm{D}$ images of pulmonary nodules and aid in transbronchial biopsy (5). However, fixed C-arm systems that perform CBCT imaging are of limited use with pulmonary intervention procedures due to their installation requirements. As an alternative, a new mobile $2 \mathrm{D} / 3 \mathrm{D}$ C-arm system that is able to carry out CBCT imaging and also fit into standard bronchoscopic suites would expand access to this technology to many more bronchoscopists. Avasarala et al. were the first group to report that a mobile $2 \mathrm{D} / 3 \mathrm{D} \mathrm{C}$-arm system-assisted procedure was safe and feasible for biopsy of peripheral solid lung lesions (6). However, studies on exploring the clinical value of mobile C-arm system for both transbronchial biopsy and ablation of GGO lesions are still absent, which are much more difficult to be localized than solid pulmonary nodules. Herein, we report our initial experience of using the mobile $\mathrm{C}$-arm system for transbronchial biopsy of a patient with multiple GGO nodules and another patient who underwent both biopsy and ablation procedure within the same bronchoscopic session. We present the following article in accordance with the CARE reporting checklist (available at https://dx.doi. org/10.21037/tlcr-21-561).

\section{Case presentation}

In the two patients diagnosed with GGO pulmonary nodules, one underwent transbronchial biopsy aided by the mobile 2D/3D C-arm system, one who underwent both transbronchial microwave ablation (MWA) procedure and transbronchial biopsy within one session. Both patients provided written informed consent. In each of the patients, a bronchoscope (BF-P290 or BF-1T260, Olympus, Tokyo, Japan) was incorporated with a guide sheath (K201 or K203, Olympus, Tokyo, Japan) and an EBUS probe (UM-S20-17S, Olympus, Tokyo, Japan) for localization and evaluation of the nodules. A mobile 2D/3D C-arm fluoroscopy system (Cios Spin, Siemens Healthineers, Erlangen, Germany) was also used for real-time mobile $2 \mathrm{D} / 3 \mathrm{D}$ imaging of the nodules. The 3D image acquisition of both patients' chest was performed using Cios Spin at the beginning of the procedure and at selected time points during the procedure. An electromagnetic navigation system (LungCare navigation system, LungCare Medical Technologies Ltd., Inc., Suzhou, China) was used for navigational guidance on selected nodules. The navigation pathway was planned based on both patients' preoperative CT scans. All procedures were performed under general anesthesia. Rapid on-site evaluation (ROSE), which is able to provide an initial assessment of effective sampling and evidence of malignant differentiation, was used for both patients' biopsy samples. For the ROSE analysis, a minimal amount of the specimen was smeared on slides, air dried and stained with Diff-Quik (BA-4100, Baso Diagnostics Inc., Zhuhai, China). The other fragments of tissue were removed in a $10 \%$ formalin solution for comprehensive pathological examination, by which the final diagnoses were confirmed.

A MWA generator (KY-2000, Canyon Medical Inc., Nanjing, China) and a 1.9-mm microwave electrode (KY2AAP-49H, Canyon Medical Inc., Nanjing, China) were used in the patient undergoing ablation. The MWA system has been reported to be feasible and safe both in both $e x$ vivo and in vivo porcine models (7).

\section{Patient 1: transbronchial biopsy only}

A 45-year-old woman with no obvious cause for her intermittent cough was admitted to the hospital for health examination. The chest CT scan detected multiple GGO nodules in both lungs. The largest mGGO measured 16.6-mm and located in the left upper lobe, was targeted for bronchoscopic examination (Figure 1). This lesion was difficult to visualize under fluoroscopy, and thus a mobile $3 \mathrm{D}$ imaging technique was used to identify the location of the lesion prior to the procedure (Figure 1A). EBUS was advanced under fluoroscopy to identify the target (Figure 1C), and the position of the EBUS probe in relation to the nodule was confirmed under mobile 3D imaging prior to the biopsy (Figure 1B). When the EBUS indicated that the lesion had been reached, the EBUS was removed, the bronchoscope and guide sheath left in situ, and then the biopsy forceps were inserted under fluoroscopic guidance 

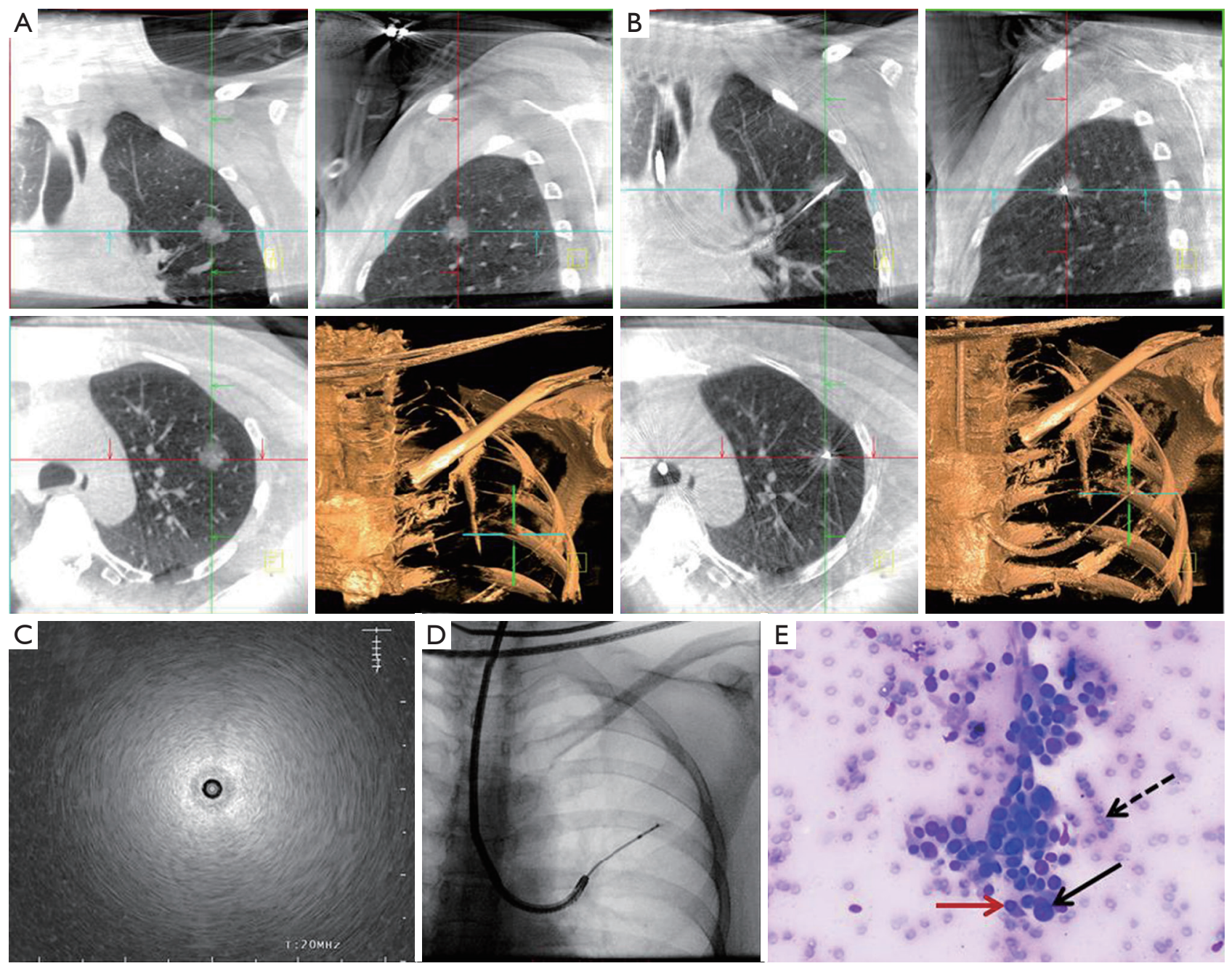

Figure 1 Transbronchial lung biopsy under guidance of mobile 3D imaging and EBUS in patient 1. (A) Pre-procedure mobile 3D imaging of the target (mGGO) confirming the location of the lesion. (B) Intra-procedure mobile 3D imaging confirmation with an EBUS probe within the target lesion. (C) Reaching the target lesion under EBUS, with "blizzard sign" observed. (D) After the insertion of biopsy forceps, transbronchial biopsy was performed under fluoroscopy. (E) In ROSE analysis, a minimal amount of the specimen was smeared on slides, air dried and stained with Diff-Quik. Adenocarcinoma was indicated in black solid arrow, red blood cells was indicated in black dotted arrow and epithelial cells was indicated in red solid arrow $(\times 400)$. mGGO, mixed ground-glass opacity; 3D, 3-dimensional; EBUS, endobronchial ultrasound; ROSE, rapid on-site evaluation.

(Figure 1D). ROSE analysis indicated adenocarcinoma (Figure 1E), which was also confirmed by the pathological examination of the biopsy sample. The procedure and image data for patient 1 are shown in Figure 1.

\section{Patient 2: transbronchial biopsy and ablation within one session}

A 71-year-old woman was diagnosed with lung adenocarcinoma based on a transbronchial biopsy seven months ago of a 15.4-mm left upper lobe GGO nodule and identified as negative in epidermal growth factor receptor (EGFR) mutation test. At the meantime, she was also found to have a 21.1-mm diameter GGO nodule in the right upper lobe. Therefore, we planned a transbronchial ablation of the left upper nodule with 3D imaging guidance, along with a transbronchial biopsy of the right upper nodule within one session in the bronchoscopy room. 

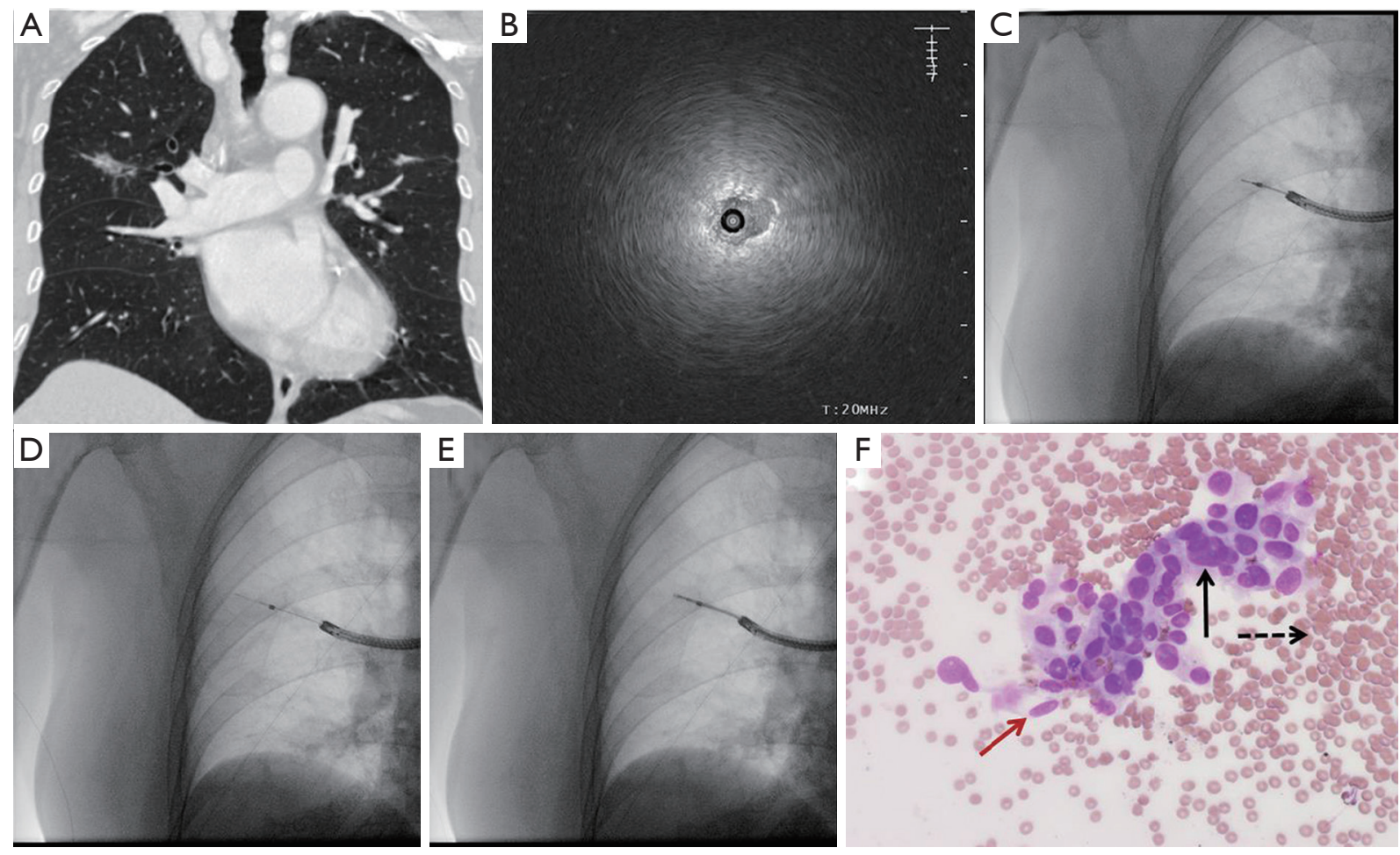

Figure 2 Transbronchial lung biopsy of the right lung in patient 2. (A) Chest CT indicated mGGO in the right upper lobe. (B) EBUS image showing a mixed blizzard sign. (C) The EBUS probe reached the lesion under fluoroscopy. (D) Transbronchial brushing under fluoroscopy. (E) Transbronchial biopsy under fluoroscopy. (F) In ROSE analysis, a minimal amount of the specimen was smeared on slides, air dried and stained with Diff-Quik. Adenocarcinoma was indicated in black solid arrow, red blood cells was indicated in black dotted arrow and epithelial cells was indicated in red solid arrow $(\times 400)$. CT, computed tomography; mGGO, mixed ground-glass opacity; EBUS, endobronchial ultrasound; ROSE, rapid on-site evaluation.

Transbronchial biopsy of the right lung lesion was performed in a manner similar fashion to that described in patient 1, guided by 2D fluoroscopy and EBUS (Figure 2). According to ROSE analysis, the lesion was identified as an adenocarcinoma. Final pathology confirmed that the lesion was malignant with EGFR mutation with deletion of exon 19 (19Del).

Immediately after the biopsy, transbronchial MWA was performed on the left lung lesion only (Figure 3). The bronchoscope with guide sheath was advanced to the target lesion with the assistance of the ENB system (Figure 3A). After confirmation with EBUS (Figure 3B), the EBUS probe was retracted and replaced with a microwave electrode. Prior to MWA treatment, a 3D imaging acquisition was performed using Cios Spin to ensure the ablating tool was positioned at the center of the lesion (Figure 3D). The ablation was then initiated at an output power of $70 \mathrm{~W}$ and continued for 8 minutes under fluoroscopy (Figure 3C).
A second mobile $3 \mathrm{D}$ scan was performed to confirm that the target lesion was covered in the ablation zone, and the increase in the size of the lesion on imaging was obvious when compared with the pre-ablation images (Figure 3E,F). No evidence of hemorrhage or pneumothorax were observed on follow-up chest CT scan the following day. A 1-month follow-up chest CT showed successful MWA with an area of thermocoagulation (Figure 3G), and a 9-month followup chest CT showed interval shrinkage and no contrast enhancement of the mass (Figure $3 H$ ).

\section{Ethics statement}

All procedures used in the studies involving human participants were performed in accordance with the ethical standards of the institutional and/or national research committee(s) and with the Helsinki Declaration (as revised in 2013). This case study was approved by the Ethics 

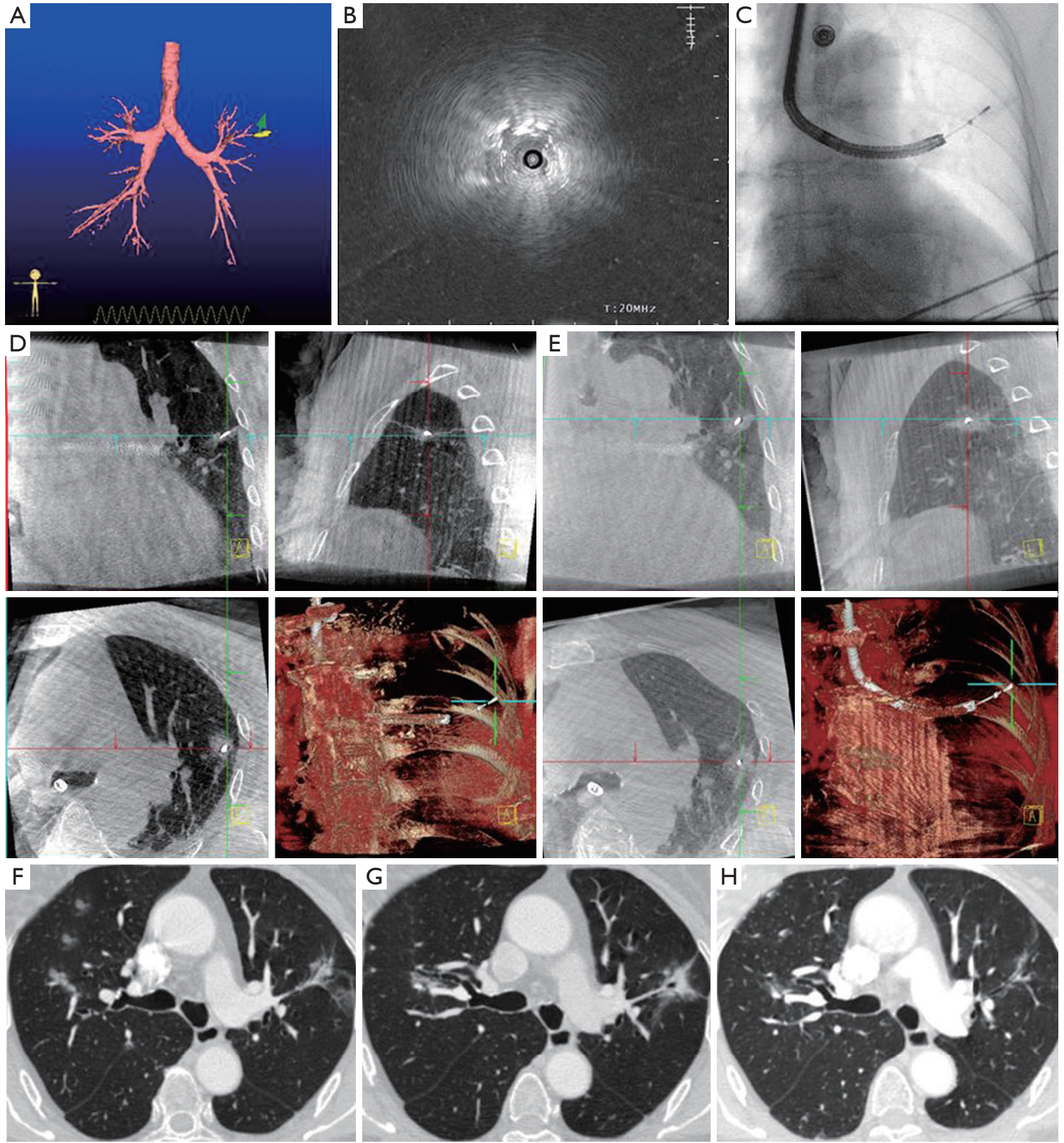

Figure 3 Transbronchial MWA of the left lung in patient 2. (A) ENB showing the locatable wire reaching the target lesion. (B) EBUS image showing a mixed blizzard sign. (C) MWA was performed under fluoroscopy. (D) Mobile 3D imaging showing the ablating tool inside of the lesion. (E) The second mobile 3D scan after ablation showing the lesion covered within the ablation area and a larger opacity, indicating satisfactory placement. (F) Chest CT target lesion before treatment. (G) One-month follow-up chest CT showing successful MWA with an area of thermocoagulation. $(\mathrm{H})$ Nine-month follow-up chest CT showing the interval shrinkage and no contrast enhancement of the mass. CT, computed tomography; MWA, microwave ablation; ENB, electromagnetic navigation bronchoscopy; EBUS, endobronchial ultrasound; 3D, 3-dimensional. 
Committee of Shanghai Chest Hospital (No. KS1813). The case of the patient who received transbronchial MWA treatment was also registered at ClinicalTrials.gov (NCT04005157).

\section{Discussion}

These two patients presented show the potential value of CBCT imaging generated by a mobile $\mathrm{C}$-arm system in visualizing GGO pulmonary nodules, evaluating "toolin-lesion" in real time, and monitoring the ablation area. In addition, the hybrid mobile 2D/3D C-arm system, combined with ENB and EBUS, expedited the diagnosis and treatment of GGO nodules, allowing for "one-stop shop" bronchoscopy feasible in a single session. Previous studies have only demonstrated the feasibility and clinical value of the mobile $2 \mathrm{D} / 3 \mathrm{D} \mathrm{C}$-arm system in biopsy procedures of peripheral lung nodules $(6,8)$. To the best of our knowledge, this is the first report of a mobile C-arm system-based CBCT imaging being used to assist in both the transbronchial biopsy and ablation of GGO lesions.

The use of CBCT in transbronchial biopsy of peripheral pulmonary nodules is an emerging field in which numerous reports have appeared (5,9-11). Hohenforst-Schmidt et al. reported that CBCT-guided bronchoscopy resulted in an overall diagnostic yield of $70 \%$, which is approximately two-fold higher than that achieved by using bronchoscopy under fluoroscopy guidance only for diagnosing incidental solitary pulmonary nodules $<2 \mathrm{~cm}$ (9). Casal et al. demonstrated that additional CBCT-guided bronchoscopy was able to increase the diagnostic yield by $20 \%$ compared to traditional thin/ultrathin bronchoscopy along with EBUS for peripheral lung nodules (10). Ali et al. reported their experience of transbronchial biopsy using an ultrathin bronchoscope guided by CBCT and VBN, which increased the overall diagnostic yield by up to $90 \%$ (11). Verhoeven et al. indicated CBCT to be a valuable addition to ENB, reporting a $35.3 \%$ increase in navigation success (12). A recent study summarized the value of CBCT imaging applied for bronchoscopy, such as instrument navigation, "tool-in-lesion" confirmation, and ablation evaluation in all phases of bronchoscopy (13). These studies suggest that CBCT has the potential to become a standard technique in this growing field.

An angio-suite equipped with a fixed C-arm system ideally could be used for CBCT-guided bronchoscopy, but such systems are typically devoted to interventional procedures by radiologists, leaving limited access for bronchoscopy. A hybrid
2D/3D mobile C-arm system was recently developed that can provide 3D CBCT imaging directly in the bronchoscopy room $(6,8)$. In this case report, we additionally evaluated the feasibility of using mobile C-arm system-based CBCT imaging in a more challenging scenario: transbronchial biopsy and ablation of GGO nodules. In contrast to solid peripheral pulmonary nodules, GGO nodules cannot be directly visualized under 2D fluoroscopy due to their unique tissue composition and thus pose a significant challenge to traditional diagnostic tools. As reported in previous studies, the diagnostic yield of transbronchial lung biopsy for GGO with guided bronchoscopy, such as EBUS and VBN, is only $57-69 \%(14,15)$. Ultrathin cryoprobe and multi-imaging modality-assisted bronchoscopic procedures are in great demand for improving the accuracy of biopsy for GGO lung nodules (16). Compared to other diagnostic methods, mobile 3D C-arm could provide real-time confirmation of "toolin-lesion", such that the device position could be adjusted accordingly based on 3D images of tool position with respect to lung nodules. This is not possible with currently used image guidance systems for lung biopsy such as EBUS or navigation bronchoscopy. In this case report, we observed that mobile C-arm system-based CBCT imaging identified GGO pulmonary nodules with satisfactory 3D image quality, potentially contributing to more accurate device positioning during the biopsy and ablation of GGO nodules.

Transbronchial ablation has recently emerged as a safe and promising therapeutic treatment for early-stage lung cancer, with fewer complications than percutaneous ablation for those patients who are not surgical candidates (17). For a more effective and safer ablation procedure for target lesions, the correct positioning of the ablation electrode at the center of the target and continuous monitoring of the ablation progress are highly valued (18). In this regard, real-time 3D imaging likely possesses greater utility and importance with the ablation procedure than with biopsy alone. This is the first case study to report the use of the mobile C-arm system-based CBCT for transbronchial ablation of a GGO lung lesion. The real-time CBCT imaging acquired using the mobile $\mathrm{C}$-arm system can allow $3 \mathrm{D}$ positioning of the ablation electrode within the lung nodule, ensuring that the focus of the ablation energy source is optimally placed centrally within the lung lesion. Another concern for transbronchial ablation is the intraprocedural assessment of the ablation effect, further confirming optimal placement of the ablation catheter. We observed that the change of the ablated lesion size was reflected in the CBCT imaging, providing real-time information for evaluating the 
endpoint of the ablation procedure. In a word, the mobile 3D C-arm system could be a promising tool for position confirmation and ablation assessment during the treatment procedures for early-stage lung cancer.

In this case study, we also explored a novel "onestop shop" workflow that was achieved by combining the biopsy and ablation of lung nodules in one session within the bronchoscopy suite. Such combination of both transbronchial biopsy, expedited diagnosis of the tissue sample, and ablation of the lesion if found to be malignant may improve the patients' experience through a reduced number of administrations of anesthetic, shortened patient waiting time between multiple procedures, decreased procedure-related anxiety, and enhance timely treatment if indicated. The required imaging guidance system for the bronchoscopic procedure varies from nodule to nodule as well as the shift from biopsy to ablation. As shown in patient 1 , the biopsy of the mGGO nodule needed imaging guidance from EBUS and real-time mobile $3 \mathrm{D}$ imaging. However, for patient 2, the biopsy procedure of an easily reachable $\mathrm{mGGO}$ nodule was performed using only $2 \mathrm{D}$ fluoroscopy and EBUS, while the ablation procedure used multiple navigation modalities, including ENB, EBUS and real-time mobile $3 \mathrm{D}$ imaging. The hybrid mobile $2 \mathrm{D} / 3 \mathrm{D}$ C-arm system may be used as an alternative system for setting-up "one-stop shop" bronchoscopy workflow, as it can also provide regular 2D fluoroscopy and comparable 3D images, allowing for pulmonologists to make appropriate 2D/3 D imaging decisions during the course of the bronchoscopy procedure.

In summary, we here present our initial experience of a hybrid mobile 2D/3D C-arm system that was used for the transbronchial biopsy and ablation of GGO nodules. Due to the limited patient cases in this study, we could demonstrate that 3D C-arm system could provide "toolin-lesion" confirmation during transbronchial access of GGO for either biopsy or ablation. Multi-modality guided bronchoscopy could be used in combination to assist in improving the accuracy and efficiency in reaching the lesion, exactly as what we actually used in the cases. Future studies with large-sized cohorts are warranted to determine the navigation accuracy, diagnostic yield and ablation efficacy utilizing real-time 3D imaging guidance from a mobile C-arm system.

\section{Acknowledgments}

The authors appreciate the academic support from AME
Interventional Pulmonology Collaborative Group.

Funding: This work was supported by National Key R\&D Program of China (no. 2017YFC0112700), the Shanghai Municipal Health and Medical Talents Training Program (no. 2018BR09), and the Shanghai Municipal Education Commission-Gaofeng Clinical Medicine Grant Support (no. 20181815).

\section{Footnote}

Reporting Checklist: The authors have completed the CARE reporting checklist. Available at https://dx.doi. org/10.21037/tlcr-21-561

Conflicts of Interest: All authors have completed the ICMJE uniform disclosure form (available at https://dx.doi. org/10.21037/tlcr-21-561). The authors have no conflicts of interest to declare.

Ethical Statement: The authors are accountable in ensuring that questions related to the accuracy or integrity of any part of the work has been appropriately investigated and resolved. All procedures used in the studies involving human participants were performed in accordance with the ethical standards of the institutional and/or national research committee(s) and with the Helsinki Declaration (as revised in 2013). Written informed consent was obtained from the patients. This case study was approved by the Ethics Committee of Shanghai Chest Hospital (No. KS1813). The case of the patient who received transbronchial MWA treatment was also registered at ClinicalTrials.gov (NCT04005157).

Open Access Statement: This is an Open Access article distributed in accordance with the Creative Commons Attribution-NonCommercial-NoDerivs 4.0 International License (CC BY-NC-ND 4.0), which permits the noncommercial replication and distribution of the article with the strict proviso that no changes or edits are made and the original work is properly cited (including links to both the formal publication through the relevant DOI and the license). See: https://creativecommons.org/licenses/by-nc-nd/4.0/.

\section{References}

1. Collins J, Stern EJ. Ground-glass opacity at CT: the ABCs. AJR Am J Roentgenol 1997;169:355-67.

2. Austin JH, Müller NL, Friedman PJ, et al. Glossary 
of terms for CT of the lungs: recommendations of the Nomenclature Committee of the Fleischner Society. Radiology 1996;200:327-31.

3. Kalanjeri S, Holladay RC, Gildea TR. State-of-the-Art Modalities for Peripheral Lung Nodule Biopsy. Clin Chest Med 2018;39:125-38.

4. Chaddha U, Kurman JS, Mahajan A, et al. Lung Nodule Management: An Interventional Pulmonology Perspective. Semin Respir Crit Care Med 2018;39:661-6.

5. Pritchett MA, Schampaert S, de Groot JAH, et al. ConeBeam CT With Augmented Fluoroscopy Combined With Electromagnetic Navigation Bronchoscopy for Biopsy of Pulmonary Nodules. J Bronchology Interv Pulmonol 2018;25:274-82.

6. Avasarala SK, Machuzak MS, Gildea TR. Multidimensional Precision: Hybrid Mobile 2D/3D C-Arm Assisted Biopsy of Peripheral Lung Nodules. J Bronchology Interv Pulmonol 2020;27:153-5.

7. Yuan HB, Wang XY, Sun JY, et al. Flexible bronchoscopyguided microwave ablation in peripheral porcine lung: a new minimally-invasive ablation. Transl Lung Cancer Res 2019;8:787-96.

8. Sadoughi A, Virdi S. Mobile 3D Intraprocedural Fluoroscopy in Combination With Ultrathin Bronchoscopy for Biopsy of Peripheral Lung Nodules. J Bronchology Interv Pulmonol 2021;28:76-80.

9. Hohenforst-Schmidt W, Zarogoulidis P, Vogl T, et al. Cone Beam Computertomography (CBCT) in Interventional Chest Medicine - High Feasibility for Endobronchial Realtime Navigation. J Cancer 2014;5:231-41.

10. Casal RF, Sarkiss M, Jones AK, et al. Cone beam computed tomography-guided thin/ultrathin bronchoscopy for

Cite this article as: Chen J, Xie F, Zheng X, Li Y, Liu S, Ma KC, Goto T, Müller T, Chan ED, Sun J. Mobile 3-dimensional (3D) C-arm system-assisted transbronchial biopsy and ablation for ground-glass opacity pulmonary nodules: a case report. Transl Lung Cancer Res 2021;10(7):3312-3319. doi: 10.21037/ tlcr-21-561 diagnosis of peripheral lung nodules: a prospective pilot study. J Thorac Dis 2018;10:6950-9.

11. Ali EAA, Takizawa H, Kawakita N, et al. Transbronchial Biopsy Using an Ultrathin Bronchoscope Guided by Cone-Beam Computed Tomography and Virtual Bronchoscopic Navigation in the Diagnosis of Pulmonary Nodules. Respiration 2019;98:321-8.

12. Verhoeven RLJ, Fütterer JJ, Hoefsloot W, et al. Cone-Beam CT Image Guidance With and Without Electromagnetic Navigation Bronchoscopy for Biopsy of Peripheral Pulmonary Lesions. J Bronchology Interv Pulmonol 2021;28:60-9.

13. Setser R, Chintalapani G, Bhadra K, et al. Cone beam CT imaging for bronchoscopy: a technical review. J Thorac Dis 2020;12:7416-28.

14. Ikezawa Y, Sukoh N, Shinagawa N, et al. Endobronchial ultrasonography with a guide sheath for pure or mixed ground-glass opacity lesions. Respiration 2014;88:137-43.

15. Ikezawa Y, Shinagawa N, Sukoh N, et al. Usefulness of Endobronchial Ultrasonography With a Guide Sheath and Virtual Bronchoscopic Navigation for Ground-Glass Opacity Lesions. Ann Thorac Surg 2017;103:470-5.

16. Jiang S, Liu X, Chen J, et al. A pilot study of the ultrathin cryoprobe in the diagnosis of peripheral pulmonary ground-glass opacity lesions. Transl Lung Cancer Res 2020;9:1963-73.

17. Harris K, Puchalski J, Sterman D. Recent Advances in Bronchoscopic Treatment of Peripheral Lung Cancers. Chest 2017;151:674-85.

18. Xie F, Zheng X, Xiao B, et al. Navigation Bronchoscopy-Guided Radiofrequency Ablation for Nonsurgical Peripheral Pulmonary Tumors. Respiration 2017;94:293-8. 